\title{
THE
}

\section{Dynamic Exponent of the Two-Dimensional Ising Model and Monte Carlo Computation of the Subdominant Eigenvalue of the Stochastic Matrix}

\author{
M. P. Nightingale \\ University of Rhode Island, nightingale@uri.edu \\ H. W.J. Blöte
}

Follow this and additional works at: https://digitalcommons.uri.edu/phys_facpubs

Terms of Use

All rights reserved under copyright.

\section{Citation/Publisher Attribution}

Nightingale, M. P., \& Blöte, H. W.J. (1996). Dynamic Exponent of the Two-Dimensional Ising Model and Monte Carlo Computation of the Subdominant Eigenvalue of the Stochastic Matrix. Physical Review Letters, 76(24), 4548-4551. doi: 10.1103/PhysRevLett.76.4548

Available at: http://dx.doi.org/10.1103/PhysRevLett.76.4548

This Article is brought to you for free and open access by the Physics at DigitalCommons@URI. It has been accepted for inclusion in Physics Faculty Publications by an authorized administrator of DigitalCommons@URI. For more information, please contact digitalcommons-group@uri.edu. 


\title{
Dynamic Exponent of the Two-Dimensional Ising Model and Monte Carlo Computation of the Subdominant Eigenvalue of the Stochastic Matrix
}

\author{
M. P. Nightingale \\ Department of Physics, University of Rhode Island, Kingston, Rhode Island 02881 \\ H. W. J. Blöte \\ Department of Applied Physics, Delft University of Technology, Lorentzweg 1, 2628 CJ Delft, The Netherlands \\ (Received 16 January 1996)
}

\begin{abstract}
We introduce a novel variance-reducing Monte Carlo algorithm for accurate determination of correlation times. We apply this method to two-dimensional Ising systems with sizes up to $15 \times 15$, using single-spin flip dynamics, random site selection, and transition probabilities according to the heat-bath method. From a finite-size scaling analysis of these correlation times, the dynamic critical exponent $z$ is determined as $z=2.1665(12)$. [S0031-9007(96)00379-1]
\end{abstract}

PACS numbers: 64.60.Ht, 02.70.Lq, 05.50.+q, 05.70.Jk

The onset of criticality is marked by a divergence of both the correlation length $\xi$ and the correlation time $\tau$. While the former divergence yields singularities in static quantities, the latter manifests itself notably as critical slowing down. To describe dynamic scaling properties, only one exponent is required in addition to the static exponents. This dynamic exponent $z$ links the divergences of length and time scales: $\tau \sim \xi^{z}$. In our computation of $z$ we exploit that, for a finite system, $\xi$ is limited by the system size $L$, so that $\tau \sim L^{z}$ at the incipient critical point.

In this Letter, we focus on the two-dimensional Ising model with Glauber-like dynamics. Values quoted in the literature for $z$ vary vastly, from $z=1.7$ to $z=2.7$ [1], but recent computations seem to be converging towards the value reported here. Finally, results are beginning to emerge of precision sufficient for sensitive tests of fundamental issues such as universality.

The numerical method introduced in this Letter is related to Monte Carlo methods used to compute eigenvalues of Hamiltonians of discrete or continuous quantum systems [2,3] and transfer matrices of statistical mechanical systems [4]. In particular, the current method is suitable to obtain more than one eigenvalue by adaptation of the diffusion Monte Carlo algorithm of Ref. [5].

To compute the correlation time of small $L \times L$ lattices we exploit the following properties of the single-spinflip Markov (or stochastic) matrix $\mathbf{P}$ [6]. It operates in the linear space of all spin configurations and its largest eigenvalue equals unity. The corresponding right eigenvector contains the Boltzmann weights of the spin configurations; the left eigenvector is constant, reflecting probability conservation. The correlation time $\tau_{L}$ (in units of one flip per spin, i.e., $L^{2}$ single-spin flips) is determined by the second-largest eigenvalue $\lambda_{L}$,

$$
\tau_{L}=-\frac{1}{L^{2} \ln \lambda_{L}} .
$$

For a system symmetric under spin inversion, the corresponding eigenvector is expected to be antisymmetric.

We used two methods to compute $\lambda_{L}$ : exact, numerical computation for $L \leq 5$ and Monte Carlo for $4 \leq L \leq 15$. The exact method used the conjugate gradient algorithm [7] and the symmetries of periodic systems. This calculation resembles that in Ref. [8], but currently we realize Glauber-like dynamics using heat-bath or Yang [9] transition probabilities and random site selection.

The Monte Carlo method used a stochastic form of the power method, as follows [5]. A spin configuration $s$ with energy $E(s)$ has a probability

$$
\frac{\exp [-E(s) / k T]}{Z} \equiv \frac{\psi_{B}(s)^{2}}{Z}
$$

where $Z$ is the partition function. The element $P\left(s^{\prime} \mid s\right)$ of the Markov matrix is the probability of a single-spinflip transition from $s$ to $s^{\prime}$. Since $\mathbf{P}$ satisfies detailed balance,

$$
\hat{P}\left(s^{\prime} \mid s\right) \equiv \frac{1}{\psi_{B}\left(s^{\prime}\right)} P\left(s^{\prime} \mid s\right) \psi_{B}(s)
$$

is symmetric. For an arbitrary trial state $|f\rangle$ an effective eigenvalue $\lambda_{L}^{(t)}$ is defined by

$$
\lambda_{L}^{(t)}=\frac{\left\langle\hat{\mathbf{P}}^{t+1}\right\rangle_{f}}{\left\langle\hat{\mathbf{P}}^{t}\right\rangle_{f}},
$$

where $\langle\cdot\rangle_{f}$ is the expectation value in the state $|f\rangle$. In the limit $t \rightarrow \infty$, the effective eigenvalue converges generically to the dominant eigenvalue allowed by the symmetry of $|f\rangle$. The convergence is exponential in the time lag $t$.

Given a trial state $|f\rangle$, standard Monte Carlo method suffices to compute the right-hand side of Eq. (4), i.e., 
the denominator of Eq. (4),

$$
\begin{aligned}
N^{(t)} & \equiv\left\langle f\left|\hat{\mathbf{P}}^{t}\right| f\right\rangle=\sum_{s_{1}, \ldots, s_{t+1}} f\left(s_{t+1}\right) \hat{P}\left(s_{t+1} 1 \mid s_{t}\right) \cdots \hat{P}\left(s_{2} \mid s_{1}\right) f\left(s_{1}\right) \\
& =\sum_{s_{1}, \ldots, s_{t+1}} \frac{f\left(s_{1}\right) f\left(s_{t+1}\right)}{\psi_{B}\left(s_{1}\right) \psi_{B}\left(s_{t+1}\right)} P\left(s_{t+1} \mid s_{t}\right) \cdots P\left(s_{2} \mid s_{1}\right) \psi_{B}\left(s_{1}\right)^{2}=Z\left\langle\frac{f\left(s_{1}\right) f\left(s_{t+1}\right)}{\psi_{B}\left(s_{1}\right) \psi_{B}\left(s_{t+1}\right)}\right\rangle_{P},
\end{aligned}
$$

is an autocorrelation; $f(s) \equiv\langle s \mid f\rangle$ and $\langle\cdot\rangle_{P}$ denotes the average with respect to the probability

$$
P\left(s_{t+1} \mid s_{t}\right) \cdots P\left(s_{2} \mid s_{1}\right) \psi_{B}\left(s_{1}\right)^{2} / Z
$$

of finding a configuration $s_{1}$ in equilibrium and subsequent transitions to configurations $s_{2}$ through $s_{t+1}$.

Similarly, the numerator of Eq. (4),

$$
\begin{aligned}
H^{(t)} & \equiv\left\langle f\left|\hat{P}^{t+1}\right| f\right\rangle \\
& =\sum_{s_{0}, \ldots, s_{t+1}} f\left(s_{t+1}\right) \hat{P}\left(s_{t+1} \mid s_{t}\right) \cdots \hat{P}\left(s_{1} \mid s_{0}\right) f\left(s_{0}\right) \\
& =\frac{1}{2} Z\left\langle\left[\lambda_{L}\left(s_{1}\right)+\lambda_{L}\left(s_{t+1}\right)\right] \frac{f\left(s_{1}\right) f\left(s_{t+1}\right)}{\psi_{B}\left(s_{1}\right) \psi_{B}\left(s_{t+1}\right)}\right\rangle_{P}
\end{aligned}
$$

is a cross correlation, where the "configurational eigenvalue" $\lambda_{L}(s)$ of spin configuration $s$ is defined as

$$
\lambda_{L}(s)=\frac{1}{f(s)} \sum_{s^{\prime}} f\left(s^{\prime}\right) \hat{P}\left(s^{\prime} \mid s\right) .
$$

Finally, with Eqs. (5) and (7), one has $\lambda_{L}^{(t)}=H^{(t)} / N^{(t)}$ for the effective eigenvalue.

In practice, $H^{(t)}$ and $N^{(t)}$ are estimated by conventional Monte Carlo methods. As usual, these estimators involve time averages of stochastic variables. Thus, on the right of Eqs. (5) and (7) $s_{i}$ is replaced by $s_{t^{\prime}+i-1}(i=1, \ldots, t)$, and the Monte Carlo average is taken over an appropriately chosen subset of times $t^{\prime}$ after thermal equilibration.

In principle, one could choose $f=m \psi_{B}$, where $m$ is the magnetization. In that case, the above method reduces to estimating the effective eigenvalue of the Markov matrix in terms of the magnetization autocorrelation function $g(t)$ via $\lambda_{L}^{(t)}=g(t+1) / g(t)$. To estimate $g(t)$ one would average over time products of the form $m\left(s_{1}\right) m\left(s_{t+1}\right)$. Equation (7) would then yield $g(t+1)$ by replacing $m\left(s_{t}\right)$ by the conditional expectation value of the magnetization at time $t+1$, evaluated explicitly as $\sum_{s_{t+1}} m\left(s_{t+1}\right) P\left(s_{t+1} \mid s_{t}\right)$.

The crux is that the estimator of $\lambda_{L}^{(t)}$ satisfies a zerovariance principle [5], since Eqs. (5) and (7) contain an optimizable trial state $|f\rangle$. In the ideal case, $|f\rangle$ is an exact eigenstate of the symmetrized Markov matrix $\hat{\mathbf{P}}$, and the "configurational eigenvalue" $\lambda_{L}(s)$ equals the eigenvalue independent of $s$. Then, the estimator of the effective eigenvalue $\lambda_{L}^{(t)}$ yields the exact eigenvalue without statistical and systematic errors at finite $t$, if care is taken to arrange cancellation of the fluctuating factors in the estimators of $H^{(t)}$ and $N^{(t)}$. It should be noted that this is true only if the numerator of Eq. (4) is evaluated with Eq. (7), in which the change from $t$ to $t+1$ is made by an explicit matrix multiplication, rather than by using the analog of Eq. (5) with $t$ replaced by $t+1$. In practice, $|f\rangle$ is not an exact eigenstate, and this introduces statistical and systematic errors. However, these errors are kept small by the zero-variance principle, if the trial states are accurate.

Such optimized trial states are constructed prior to the main Monte Carlo run, by minimization of the variance $\chi^{2}$ of the configurational eigenvalue

$$
\chi^{2}(p)=\left\langle\left(\hat{\mathbf{P}}-\langle\hat{\mathbf{P}}\rangle_{f}\right)^{2}\right\rangle_{f} .
$$

As indicated, the variance depends on the parameters $p$ of the trial state. Optimization over $p$ is done following Umrigar, Wilson, and Wilkins [10]: one samples $M$ configurations $s_{i}$, typically a few thousand, with probability $\psi_{B}^{2} Z^{-1}$ and approximates $\chi^{2}(p)$ by

$$
\chi^{2}(p) \approx \frac{\sum_{i=1}^{M}\left[f\left(s_{i}, p\right) / \psi_{B}\left(s_{i}\right)\right]^{2}\left[\lambda_{L}\left(s_{i}, p\right)-\bar{\lambda}_{L}(p)\right]^{2}}{\sum_{i=1}^{M}\left[f\left(s_{i}, p\right) / \psi_{B}\left(s_{i}\right)\right]^{2}} .
$$

Here $\bar{\lambda}_{L}$ denotes the weighted average of the configurational eigenvalue over the sample, while the modified notation explicitly shows dependences on the parameters $p$ of the trial state $|f\rangle$. Near-optimal values of the parameters $p$ can be obtained by minimization of the expression on the right-hand side of Eq. (10) for a fixed sample. Statistical independence in the sample requires that the configurations be selected at intervals on the order of the correlation time.

A guiding principle for the construction of trial states is that long-wavelength fluctuations of the magnetization have the longest decay time. Furthermore, analysis of the exact left eigenvectors of the Markov matrix $\mathbf{P}$ for systems with $L \leq 5$ shows that the elements depend only on the magnetization to good approximation. This suggests trial functions depending on long-wavelength components of the Fourier transform of $s_{i}$, the zeromomentum component of which is just the magnetization $m$. The form

$$
f(s)=\tilde{\psi}_{B}(s) \psi^{(+)}(s) \psi^{(-)}(s),
$$

where $\psi^{( \pm)} \rightarrow \pm \psi^{( \pm)}$under spin inversion, yields an antisymmetric trial function, as required. The tilde in 
$\tilde{\psi}_{B}$ indicates that the temperature is used as a variational parameter, but we found that its optimal value is virtually indistinguishable from the true temperature. The $\psi^{( \pm)}$ were chosen as

$$
\begin{aligned}
& \psi^{(+)}=\sum_{\mathbf{k}} a_{\mathbf{k}}\left(m^{2}\right) m_{\mathbf{k}}^{(+)}+m \sum_{\mathbf{k}} b_{\mathbf{k}}\left(m^{2}\right) m_{\mathbf{k}}^{(-)}, \\
& \psi^{(-)}=m \sum_{\mathbf{k}} c_{\mathbf{k}}\left(m^{2}\right) m_{\mathbf{k}}^{(+)}+\sum_{\mathbf{k}} d_{\mathbf{k}}\left(m^{2}\right) m_{\mathbf{k}}^{(-)},
\end{aligned}
$$

where the index $\mathbf{k}$ runs through a small set of multiplets of four or fewer long-wavelength wave vectors defining the $m_{\mathbf{k}}^{( \pm)}$, translation and rotation symmetric sums of products of Fourier transforms of the local magnetization; the $\mathbf{k}$ are selected so that $m_{\mathbf{k}}^{(-)}$is odd and $m_{\mathbf{k}}^{(+)}$is even under spin inversion; the coefficients $a_{\mathbf{k}}, b_{\mathbf{k}}, c_{\mathbf{k}}$, and $d_{\mathbf{k}}$ are polynomials of second order or less in $m^{2}$. The degrees of these polynomials were chosen so that no terms occur of higher degree than four in the spin variables. We used trial functions dependent on system size only in the optimal values of the parameters. This yielded a $\chi^{2}$ and an error in the variational estimate $\lambda_{L}^{(1)}$ decreasing with $L$; yet, the relative error in $\tau_{L}$ increases.

Since the probability distribution Eq. (6) is precisely the one purportedly generated by standard Monte Carlo method, the sampling procedure is straightforward. The Monte Carlo algorithm used a random-number generator of the shift-register type. It was selected with care to avoid the introduction of systematic errors; see discussion and references in Ref. [11]. We used two KirkpatrickStoll [12] generators, the results of which were combined by a bitwise exclusive or [13]. For test purposes we replaced one Kirkpatrick-Stoll generator by a linear congruential rule, but this did not reveal clear differences [11].

For each system size $4 \leq L \leq 15$, Monte Carlo averages were taken over $8 \times 10^{8}$ spin configurations. For $L=13-15$ these were separated by intervals of 16 sweeps (Monte Carlo steps per spin); 8 sweeps for $L=11$ and 12; 2 sweeps for $L=5$ and 6; and only one sweep for $L=4$. The simulations of the remaining system sizes consisted of parts using intervals of 2,4 , or 8 sweeps.

The numerical results for the effective second largest eigenvalue $\lambda_{L}^{(t)}$ as a function of the projection time $t$ appeared to converge rapidly. In agreement with scaled results for $L \leq 5$ spectra, we observe that convergence occurs within a few intervals as given above. Monte Carlo estimates of $\lambda_{L}$ are shown in Table I, as are exact results for small systems. For system sizes $L=4$ and 5, the two types of calculation agree satisfactorily. The small numerical errors indicate that the variance-reducing method introduced above is quite effective.

For finite system size $L$ there are corrections to the leading scaling behavior $\tau_{L} \sim L^{z}$. In the twodimensional Ising model corrections to static equilibrium quantities occur with even powers of $1 / L[14]$; thus we
TABLE I. Second-largest eigenvalue $\lambda_{L}$ of the Markov matrix. The first column indicates the method: exact numerical or Monte Carlo.

\begin{tabular}{lrcc}
\hline \hline Method & $L$ & $\lambda_{L}$ & Error \\
\hline Exact & 2 & 0.985702260395516 & 0.000000000001 \\
Exact & 3 & 0.997409385126011 & 0.000000000001 \\
Exact & 4 & 0.999245567376453 & 0.000000000001 \\
Exact & 5 & 0.999708953624452 & 0.000000000001 \\
MC & 4 & 0.9992455685 & 0.0000000094 \\
MC & 5 & 0.9997089453 & 0.0000000060 \\
MC & 6 & 0.9998657194 & 0.0000000045 \\
MC & 7 & 0.9999299708 & 0.0000000031 \\
MC & 8 & 0.9999600854 & 0.0000000023 \\
MC & 9 & 0.9999756630 & 0.0000000017 \\
MC & 10 & 0.9999843577 & 0.0000000014 \\
MC & 11 & 0.9999895056 & 0.0000000010 \\
MC & 12 & 0.9999927107 & 0.0000000008 \\
MC & 13 & 0.9999947840 & 0.0000000006 \\
MC & 14 & 0.9999961736 & 0.0000000005 \\
MC & 15 & 0.9999971314 & 0.0000000005 \\
\hline \hline
\end{tabular}

expect

$$
\tau_{L} \approx L^{z} \sum_{k=0}^{n_{c}} \alpha_{k} L^{-2 k}
$$

where the series was arbitrarily truncated at order $n_{c}$, but other powers of $1 / L$ might occur as well. Ignoring the latter, we fitted the correlation times of Table I to this form. Typical results of such fits are given in Table II. The smallest systems do not fit Eq. (14) well, at least not for the $n_{c}$ values used. The residuals decrease rapidly

TABLE II. Results of least-squares fits for the dynamic exponent. The first column shows the minimum system size included, the second the number of correction terms included, and the third column whether (y) or not (n) numerical exact results (for $L \leq 5$ ) are included. The last column contains the chi-square confidence index [16].

\begin{tabular}{cccccc}
\hline \hline$L \geq$ & $n_{c}$ & Exact & $z$ & Error & $Q$ \\
\hline 4 & 1 & $\mathrm{n}$ & 2.1769 & 0.0001 & 0.00 \\
5 & 1 & $\mathrm{n}$ & 2.1705 & 0.0002 & 0.00 \\
6 & 1 & $\mathrm{n}$ & 2.1688 & 0.0003 & 0.23 \\
7 & 1 & $\mathrm{n}$ & 2.1679 & 0.0006 & 0.43 \\
8 & 1 & $\mathrm{n}$ & 2.1672 & 0.0010 & 0.42 \\
4 & 2 & $\mathrm{n}$ & 2.1650 & 0.0003 & 0.17 \\
5 & 2 & $\mathrm{n}$ & 2.1665 & 0.0006 & 0.70 \\
6 & 2 & $\mathrm{n}$ & 2.1662 & 0.0013 & 0.60 \\
7 & 2 & $\mathrm{n}$ & 2.1648 & 0.0024 & 0.52 \\
4 & 3 & $\mathrm{n}$ & 2.1672 & 0.0009 & 0.64 \\
5 & 3 & $\mathrm{n}$ & 2.1656 & 0.0020 & 0.61 \\
6 & 3 & $\mathrm{n}$ & 2.1625 & 0.0044 & 0.56 \\
3 & 3 & $\mathrm{y}$ & 2.1653 & 0.0004 & 0.34 \\
4 & 3 & $\mathrm{y}$ & 2.1670 & 0.0009 & 0.64 \\
5 & 3 & $\mathrm{y}$ & 2.1657 & 0.0020 & 0.49 \\
\hline \hline
\end{tabular}


TABLE III. Comparison of recent results for the dynamic exponent $z$. Numerical errors are in parentheses.

\begin{tabular}{lll}
\hline \hline \multicolumn{1}{c}{ Reference } & Year & \multicolumn{1}{c}{ Value } \\
\hline Present work & 1996 & $2.1665(12)$ \\
Li et al. [15] & 1995 & $2.1337(41)$ \\
Linke et al. [17] & 1995 & $2.160(5)$ \\
Grassberger [18] & 1995 & $2.172(6)$ \\
Wang et al. [19] & 1995 & $2.16(4)$ \\
Baker and Erpenbeck [20] & 1994 & $2.17(1)$ \\
Ito [21] & 1993 & $2.165(10)$ \\
Dammann and Reger [22] & 1993 & $2.183(5)$ \\
Matz et al. [23] & 1993 & $2.35(5)$ \\
Münkel et al. [24] & 1993 & $2.21(3)$ \\
Stauffer [25] & 1993 & $2.06(2)$ \\
\hline \hline
\end{tabular}

when the minimum system size is increased and the consistency between the results for different $n_{c}$ suggests that Eq. (14) captures the essential scaling behavior of $\tau_{L}$. From these results we chose the entry for $L \geq 5$ and $n_{c}=$ 2 as our best estimate: $z=2.1665 \pm 0.0012$, where we conservatively quote a $2 \sigma$ error. To our knowledge, this is the most precise estimate of $z$ obtained to date, as evidenced by recent results summarized in Table III. The table shows that the mutual consistency of the results for $z$ has tended to improve in recent years. The only recent result that appears inconsistent with ours is due to Li, Schulke, and Zheng [15]. Its error is copied from Table $\mathrm{I}$ of $\mathrm{Li}$ et al. The data in that table display finitesize dependences that exceed the quoted errors, which may explain the discrepancy with our result.

This research was supported by the (U.S.) National Science Foundation through Grant No. DMR-9214669, by the Office of Naval Research and by the NATO through Grant No. CRG 910152. This research was conducted in part using the resources of the Cornell Theory Center, which receives major funding from the National Science Foundation (NSF) and New York State, with additional support from the Advanced Research Projects Agency (ARPA), the National Center for Research Resources at the National Institutes of Health (NIH), IBM Corporation, and other members of the center's Corporate Research Institute.

[1] See, e.g., references in G.F. Mazenko and O.T. Valls, Phys. Rev. B 24, 1419 (1981), and in M.-D. Lacasse, J. Viñals, and M. Grant, Phys. Rev. B 47, 5646 (1993).

[2] S. Zhang, N. Kawashima, J. Carlson, and J. E. Gubernatis, Phys. Rev. Lett. 74, 1500 (1995), and references therein.

[3] M. Takahashi, Phys. Rev. Lett. 62, 2313 (1989).
[4] M.P. Nightingale, E. Granato, and J. M. Kosterlitz, Phys. Rev. B 52, 7402 (1995); M.P. Nightingale and H. W. J. Blöte, Phys. Rev. B (to be published), and references therein.

[5] D. M. Ceperley and B. Bernu, J. Chem. Phys. 89, 6316 (1988); B. Bernu, D. M. Ceperley, and W. A. Lester, J. Chem. Phys. 93, 552 (1990).

[6] See, e.g., W. Feller, An Introduction to Probability Theory and its Applications (John Wiley and Sons, New York, 1968), Vol. 1; H. Haken, Synergetics: an Introduction: Nonequilibrium Phase Transitions and Self-Organization in Physics, Chemistry, and Biology (Springer-Verlag, Berlin, 1978).

[7] M. P. Nightingale, V.S. Viswanath, and G. Müller, Phys. Rev. B 48, 7696 (1993).

[8] M.P. Nightingale and H. W. J. Blöte, Physica (Amsterdam) 104A, 352 (1980).

[9] C. P. Yang, Proc. Symp. Appl. Math. 15, 351 (1963).

[10] C. J. Umrigar, K. G. Wilson, and J. W. Wilkins, Phys. Rev. Lett. 60, 1719 (1988); in Computer Simulation Studies in Condensed Matter Physics: Recent Developments, edited by D. P. Landau, K. K. Mon, and H. B. Schüttler, Springer Proceedings of Physics Vol. 33 (Springer, Berlin, 1988); C. J. Umrigar, Int. J. Quant. Chem. Symp. 23, 217 (1989).

[11] H. W. J. Blöte, E. Luijten, and J. R. Heringa, J. Phys. A 28, 6289 (1995).

[12] S. Kirkpatrick and E. P. Stoll, J. Comput. Phys. 40, 517 (1981).

[13] J. R. Heringa, H.W. J. Blöte, and A. Compagner, Int. J. Mod. Phys. C 3, 561 (1992).

[14] See, e.g., H. W. J. Blöte and M. P. M. den Nijs, Phys. Rev. B 37, 1766 (1988), and references therein.

[15] Z. B. Li, L. Schulke, and B. Zheng, Phys. Rev. Lett. 74, 3396 (1995).

[16] W.H. Press, B.P. Flannery, S. A. Teukolsky, and W. T. Vetterling, Numerical Recipes (Cambridge University Press, Cambridge, 1986 and 1992), Sec. 6.2.

[17] A. Linke, D. W. Heermann, P. Altevogt, and M. Siegert, Physica (Amsterdam) 222A, 205 (1995).

[18] P. Grassberger, Physica (Amsterdam) 214A, 547 (1995).

[19] F. Wang, N. Hatano, and M. Suzuki, J. Phys. A 28, 4543 (1995).

[20] G. A. Baker, Jr. and J.J. Erpenbeck, in Computer Simulation Studies in Condensed Matter Physics, edited by D. P. Landau, K. K. Mon, and H. B. Schüttler, Springer Proceedings in Physics Vol. 78 (Springer-Verlag, Berlin, 1994), Vol. VII.

[21] N. Ito, Physica (Amsterdam) 196A, 591 (1993).

[22] B. Dammann and J.D. Reger, Europhys. Lett. 21, 157 (1993).

[23] R. Matz, D. L. Hunter, and N. Jan, J. Stat. Phys. 74, 903 (1993).

[24] C. Münkel, D. W. Heermann, J. Adler, M. Gofman, and D. Stauffer, Physica (Amsterdam) 193A, 540 (1993).

[25] D. Stauffer, J. Phys. A 26, L599 (1993). 